\title{
Clinical Profile of Severe Cutaneous Drug Eruptions in a Tertiary Care Hospital
}

\author{
Rupak Bishwokarma Ghimire, ${ }^{1}$ Sabina Bhattarai, ${ }^{1}$ Govinda Pokharel, ${ }^{1}$ Eliz Aryal, ${ }^{1}$ Prashanna Raj \\ Shrestha ${ }^{1}$ \\ ${ }^{1}$ Department of Dermatology, Kathmandu Medical College, Kathmandu, Nepal.
}

\begin{abstract}
Background: An adverse cutaneous reaction caused by a drug is any undesirable change in the structure or function of the skin, its appendages or mucous membranes and it encompass all adverse events related to drug eruption, regardless of the etiology. Methods: This is a cross-sectional study done from April 2017 to March 2019 at dermatology department of Kathmandu Medical College Teaching Hospital. Sample size was calculated as 42 with prevalence of severe cutaneous drug eruption as 3\%. After the medication history was taken, all suspected causative drugs were discontinued. For the initial 5 to 7 days, all patients were treated with intravenous corticosteroids and oral antihistamines. Follow up after one week, 2 weeks and one month were suggested for assessment of outcomes. Results: Out of 42 patients, $22(52.38 \%)$ were females and 20 $(47.62 \%)$ males. Most were in age groups 16-39 \& 46-60 counting to $14(33.33 \%)$ in each group. Acute morbilliform eruption was the most common presentation $20(47.6 \%)$ followed by Steven Johnson Syndrome -Toxic Epidermal Necrolysis in 16 (38.1\%). The most common offending drug group in this study was antileptics $9(21.43 \%)$, followed by non-steroid anti inflammatory drugs NSAIDs 8 (19.05\%), antibiotics 7 $(16.67 \%)$, allopurinol $4(9.52 \% \%)$. Patients were discharged after 7 days with tapering dose of oral prednisolone upto 6 weeks for all patients. However, three patients died due to Toxic Epidermal Necrolysis along with comorbid conditions like intracerebral hematoma, COPD with pneumonia. Conclusions: Adverse cutaneous drug reactions should be recognized early for timely intervention. Previously sensitized patients should be made aware about fatal outcomes, symptoms, cross reactivites as well as over the counter preparations.
\end{abstract}

Keywords: adverse cutaneous drug reactions; drug hypersensitivity.

\section{INTRODUCTION}

An adverse cutaneous drug reaction (ACDR) is any undesirable change in the structure or function of the skin, its appendages or mucous membranes and it encompass all adverse events related to drug eruption, regardless of the etiology. ${ }^{1}$ According to the World Health Organization, Severe Cutaneous Adverse Reactions to Drugs (SCARD) are those resulting in death, requiring hospitalization or those that extend the length of stay, result in persistent or significant disability or are life threatening. ${ }^{2}$

ACDRs are frequent, affecting 2-3\% of all hospitalized patients but only $2 \%$ are severe and very few are fatal. ${ }^{3}$ Numerous cutaneous reaction patterns have been reported including acute exanthematous eruptions, urticaria, angioedema, bullous eruptions, acute exanthematous generalised pustulosis (AGEP), fixed drug eruptions (FDE), erythema multiforme, pruritus, and life-threatening cutaneous reactions such as Stevens-Johnson syndrome (SJS), toxic epidermal necrolysis (TEN), exfoliative dermatitis, and drug rash with eosinophilia and systemic symptoms (DRESS). ${ }^{4}$ The objective of this study was to assess the clinical presentations and response to intravenous steroid therapy to cutaneous drug eruptions.

\section{METHODS}

This is a cross-sectional study done fom April 2017 to March 2019 at dermatology department of Kathmandu Medical College Teaching Hospital. Ethical clearance was taken from Institutional Review Committee (IRC) Ref 200520191. Sample size was calculated as 42 with prevalence of severe cutaneous drug eruption as 3\% with margin of error $5 \%$ using the formula. ${ }^{4}$

$\mathrm{N}=$ Confidence Interval $(\mathrm{CI})^{2} \times \mathrm{P} \times(1-\mathrm{P}) / \mathrm{M}^{2}$.

We evaluated demographic characteristics, offending drugs, latency period, laboratory results, response to treatment, and outcome. The offending drugs were suspected by medication history and clinical course of skin eruptions, and diagnosis confirmed by at least two dermatologists. Complete blood counts, liver function tests, determination of serum creatinine levels, and urinalyses were performed to identify internal

Correspondence: Dr. Rupak Bishwokarma Ghimire, Department of Dermatology, Kathmandu Medical College, Sinamangal, Kathmandu, Nepal. Email: rupakghi@gmail.com. Phone: +977-9802900007. Article Received: 2019-0303. Article Accepted: $2019-8-16$. 
organ involvements. Specific drug allergy and offending drugs were suspected following the NICE guidelines. ${ }^{5}$

After the medication history was taken, all suspected causative drugs were discontinued. For the initial 5 to 7 days, all patients were treated with intravenous corticosteroids dexamethasone $8 \mathrm{mg}$ per day and oral antihistamines and prophylactic antibiotics. Treatment response was assessed by healing of skin lesions, daily monitoring of vitals, urinary output. Repeat of complete blood counts, liver function test, serum creatinine levels, and urinalyses were done before discharge.

Oral Prednisolone was administered at $0.5-1.0 \mathrm{mg} /$ $\mathrm{kg}$ along with oral antihistamines on discharge to prevent flare up after stopping intravenous steroids and follow up after one week, 2 weeks and six weeks were suggested to all patients. Patients who lost followed up were contacted through telephone at the end of six weeks. Re-challenge test was done for antitubercular drugs according to National Tuberculosis Drug Protocol of Nepal. Statistical analyses were performed using SPSS version 22 (SPSS Inc, Chicago, Illinois, USA).

\section{RESULTS}

Out of 42 patients, $22(52.38 \%)$ of the patents were females and $20(47.62 \%)$ males with slight female preponderance. Mean age was 39.85 years. Majority of the patients were in age groups 16-39 \& ages $46-60$ counting to $14(33.33 \%)$ in each groups respectively, third common age group

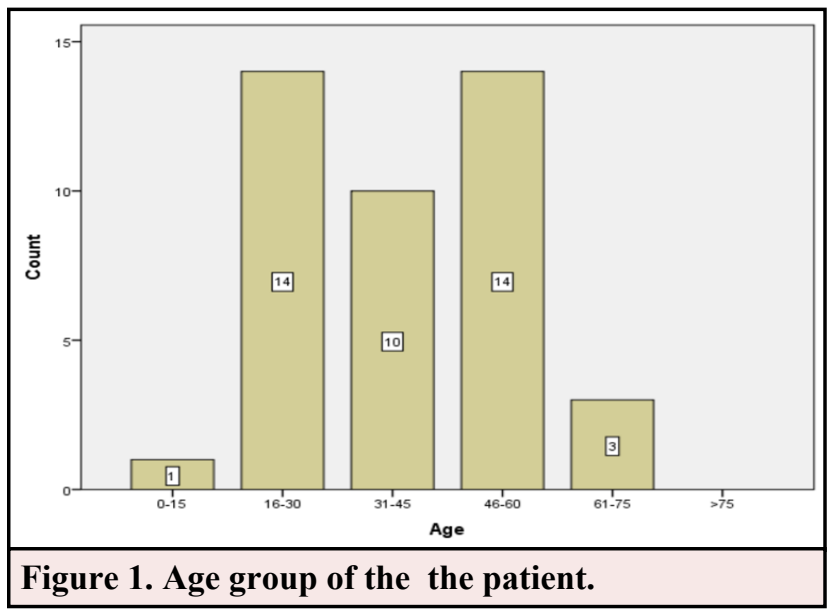

being from ages $31-45$ as $10(23.8 \%)$. Only 3 $(1.5 \%)$ patients were in age group $61-75 \&$ one patient was 13 years old, in age group less than 15 years as depicted in Figure 1. Acute morbilliform eruption was the most common diagnosis occurring in $20(47.6 \%)$ followed by SJS/TEN in $16(38.1 \%)$ patients. Urticarial lesions were seen in $3(7.14 \%)$, DRESS syndrome, AGEP and FDE were in one patient each, shown in Figure 2. The most common offending drug in this study was

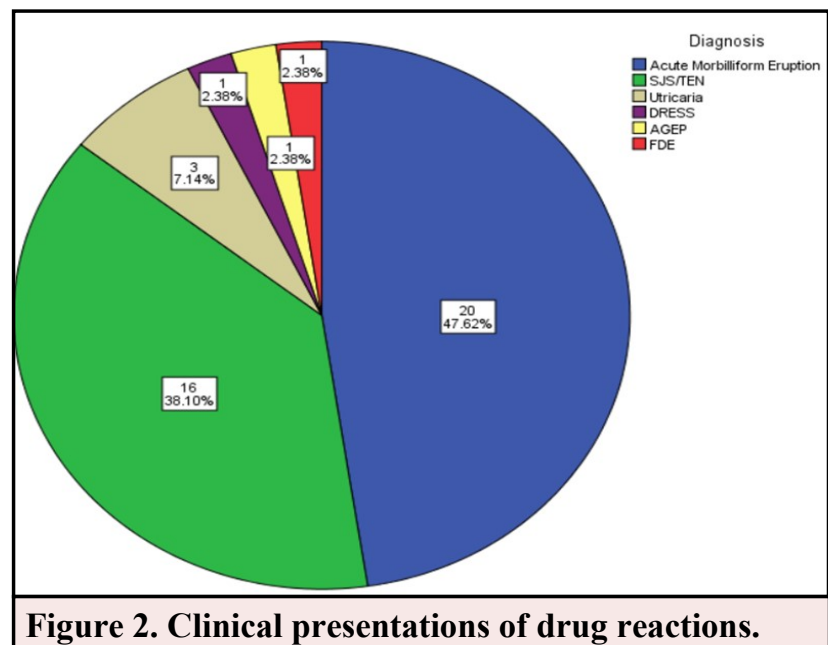

antileptics $9(21.43 \%)$, followed by non-steroidal anti inflammatory drugs (NSAIDs) in $8(19.05 \%)$, antibiotics 7 (16.67\%), allopurinol $4(9.52 \% \%)$. antitubercular treatment (ATT) were involved in 2 (4.76\%) patients out of which Rifampicin was identified as offending drug after re-challenge test in one patient and exact drug couldn't be identified in another patient. Hydroxychloroquine, methotrexate, sulfasalazine, rifampicin were involved in one patient each. Ayurvedic medicine with no identified name was suspected in one patient. In seven (16.67\%) patients, single drug couldn't be elicited as they had taken multiple drugs before the reaction occurred summarized in

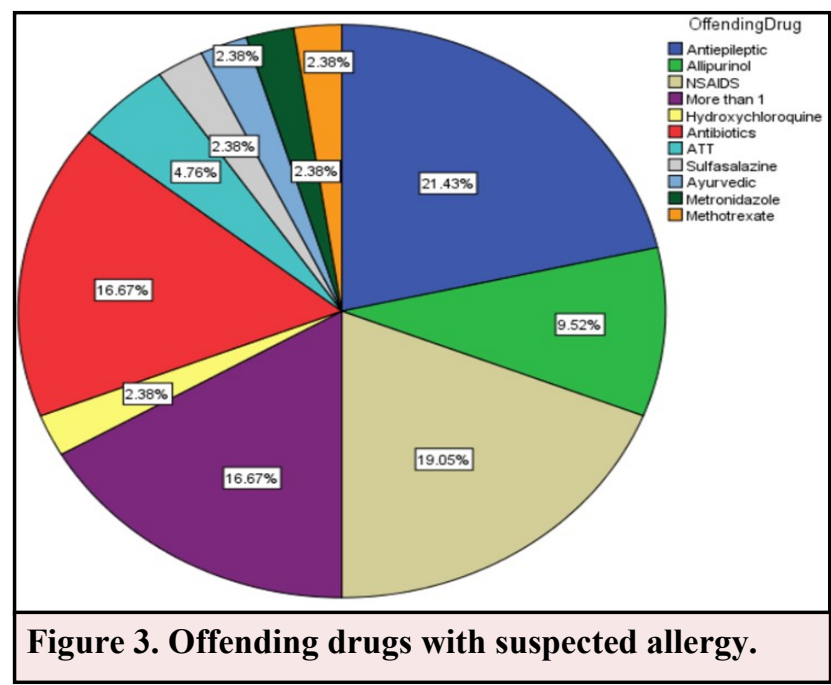

Figure 3. Among nine patients with offending drugs as anticonvulsants, Phenytoin was the commonest drug in five patients followed by lamotrigine in three and carbamazepine in one patient. Duration of reactions after the intake of drugs ranged from immediately within five minutes to six weeks after with a mean duration of 10.16 days.

Most of the patients recovered well and discharged after 7 days with tapering dose of oral prednisolone 
upto 6 weeks. However, three patients died. One patient died with SJS/TEN on repeated exposure to NSAIDs, instead of the fact that she was warned about avoidance of related medicines. Another patient died of TEN with NSAIDs along with comorbidity of intracerebral hematoma. Third patient died with TEN with chronic obstructive pulmonary disease (COPD) and pneumonia with suspected allergy to multiple drugs including Azithromycin, NSAIDs.

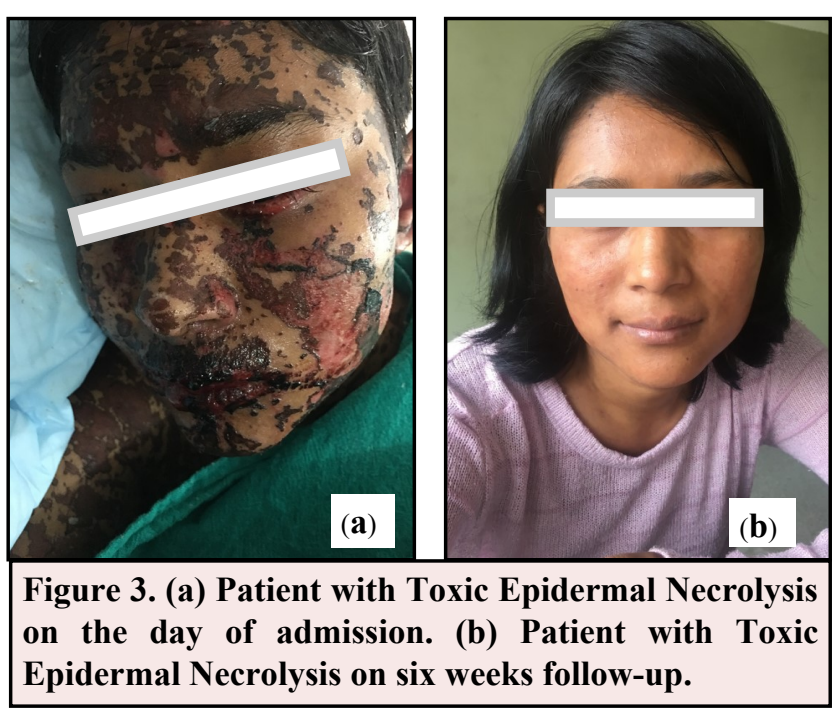

\section{DISCUSSION}

Many studies from different parts of the world have shown similar findings like our study, with slight differences in percentages of epidemiological and clinical presentations.

The finding from our study showed a slight female preponderance $22(52.38 \%)$. Sonntag et al noted that higher female-male ratio may have been by greater drug consumption by women. ${ }^{6}$ Whereas in a study carried out in India with a similar sample size like ours with 43 cases of severe cutaneous drug reactions, majority was in age group 46-60 years with male preponderance SJS-TEN being the commonest reaction pattern followed by maculopapular rash and DRESS. Commonest drug group producing reactions were aromatic anticonvulsants $20(46.5 \%)$ followed by antibiotics and NSAIDs. Phenytoin was the commonest offender. ${ }^{7}$ In contrary, acute morbilliform eruptions were commonest reaction pattern in our study followed by SJS-TEN. Commonest offending drugs were anticonvulsants, mainly phenytoin as the commonest offender in our study as well.

In a one-year study from India, with a smaller sample size of 30 patients with cutaneous drug reactions, 15 patients required hospitalization. Of these, three patients had toxic epidermal necrolysis, 3 patients had Stevens-Johnson syndrome, 8 patients had exfoliative dermatitis and 1 patient had fixed drug eruption. Commonest drugs producing reactions were amoxicillin $(23 \%)$, paracetamol $(16 \%)$, carbamazepine $(10 \%)$ and phenytoin $(10 \%)$ quite different from our study where anticonvulsants were the commonest offending drugs. Four patients had consumed the same drug earlier and developed similar reaction like toxic epidermal necrolysis with amoxicillin, fixed drug eruption with ciprofloxacin, TEN with sodium valproate. ${ }^{8}$ Similar history was seen in two of our patients - one developed toxic epidermal necrolysis and other had fixed drug eruption.

Out of 106 patients admitted of ACDR in Turkey from 2002 to 2009, 71 females and 35 males were evolved. Mean age was 44.03 slightly higher than the mean age of our study which was 39.85 years. Duration between drug intake and onset of reaction varied from 5 minutes to 3 months. The most common clinical type was urticaria and/or angioedema in $48.1 \%$ of the patients, followed by maculopapular rash in $13.2 \%$, and DRESS in $8.5 \%$. Drugs most frequently associated with cutaneous drug eruptions were antimicrobial agents in $40.5 \%$ of the patients, followed by antipyretic/antiinflammatory analgesics in $31.1 \%$, and antiepileptics in $11.3 \%{ }^{9}$ These findings have slight differences in offending drugs and clinical presentations from our study.

A similar two-year study was carried out in Malaysia with a larger sample size of 134 patients. The mean age for the study population was 47 years, the youngest being 14 and the oldest 91 years. The majority $71.6 \%$ of cases were 59 years and below. There were no patients below the age of 12 years. Males and females were equally affected (1.1:1). The commonest manifestations of ACDR include maculopapular rash in $30(22.4 \%)$ cases followed by SJS in $13(9.7 \%)$ cases. ${ }^{10}$

There are not many previous studies that looked at the relationship between duration of drug intake and onset of ACDR. Our study showed duration of reactions after the intake of drugs ranged from immediately within five minutes to six weeks after with a mean duration of 10.16 days. A study from Turkey showed that the mean time duration between drug consumption and initial reaction was 2 weeks with a wide range between 5 minutes and 120 days. $^{8}$ The onset of reaction is determined by drug and host factors. The chemical compound, physical form, half-life and the immune response triggered by the drug influences the onset of adverse reactions. ${ }^{11}$

A larger five years retrospective study was carried out in in Brazil where out of 298 patients diagnosed with cutaneous drug reactions, 173 patients were admitted in hospital. Ages of the patients were 
ranging from one month to 60 years and median age being 38.4 years quite similar to our study. In cases where the cause was determined, the most common suspected drug class was anticonvulsants $40.4 \%$, followed by antibiotics $22.8 \%$, whereas analgesics and anti-inflammatory drugs accounted for 6 cases of SCARD with similar findings like our study. In 10 patients $(17.5 \%)$, the drug causing the cutaneous drug reactions could not be determined similar to our findings where single offending drug category couldn't be identified in $7(16.67 \%)$ cases. Of the SCARD patients, $10(17.5 \%)$ died which was similar to our study where $7.1 \% \quad(n=3)$ patients died. $^{12}$

The death of or patients may have been attributed to co existing diseases as well than merely due to isolated drug reactions as the primary cause.

\section{REFERENCES}

1. Nayak S, Acharjya B. Adverse Cutaneous Drug Reaction. Indian J Dermatol. 2008; 53(1): 2-8. https://doi.org/10.4103/0019-5154.39732 PMid:19967009 PMCid:PMC2784579

2. Revuz J, Valeyrie-Allanore L. Drug Reactions. In: Bolognia JL, Jorizzo JL, Rapini RP, editors. Dermatology. 2nd ed. Madrid, Spain: Mosby; 2008. 331-20.

3. Wiffen P, Gill M, Edwards J, Moore A. Adverse drug reactions in hospital patients: A systematic review of the prospective and retrospective studies. Bandolier Extra. 2002:116. [Google Scholar | Full Text ]

4. McKenna JK, Leiferman KM. Dermatologic drug reactions. Immunol Allergy Clin North Am. 2004; 24:399-423. https://doi.org/10.1016/ j.iac.2004.03.007 PMid:15242718.

5. NICE. Drug allergy: diagnosis and management. NICE quality standard 97 . NICE, 2015: 34-35. Available at: https:// www.nice.org.uk/guidance/cg183/evidence/fullguideline-pdf-193159693

6. Sonntag MR, Zoppi M, Fritschy D et al. Exanthema during frequent use of antibiotics and antibacterial drugs (penicillin, especially aminopenicillin, cephalosporin and cotrimoxazole) as well as allopurinol: results of the Berne Comprehensive Hospital Drug Monitoring program. Schweiz Med Wochenshr. 1986;116:142- 145
However, it has to be kept in mind that drug reactions can lead to fatal outcomes especially in patients with re-exposure to drugs.

Limitation of our study is that it was a single hospital based study, which may require multicenter study with larger sample size.

\section{CONCLUSIONS}

Adverse cutaneous drug reactions are alarming to both patients and treating doctors, should be recognized early for timely intervention. Previously sensitized patients should be made aware about fatal outcomes, potential drugs, symptoms of drug reactions, cross reactivities as well as over the counter preparations.

\section{Conflict of Interest: None}

7. Sasidharanpillai S, Riyaz N, Khader A, Rajan U, Binitha MP, Sureshan DN. Severe cutaneous adverse drug reactions: a clinicoepidemiological study. Indian J Dermatol. 2015; 60(1):102 https://doi.org/10.4103/0019-5154.147834 PMid:25657416 PMCid:PMC4318022

8. Ahamed RNN, Swaminathan A, Priyadarshini A, et al. Pattern of adverse cutaneous drug reactions in a tertiary care hospital. J. Evolution Med. Dent. Sci. 2017; 6(89):6237-40 |https:// doi.org/10.14260/jemds/2017/1356

9. Akpinar F, Dervis E. Drug eruptions: an 8-year study including 106 in patients at a dermatology clinic in Turkey. Indian J Dermatol. 2012; 57 (3): 194-8. https://doi.org/10.4103/00195154.96191 PMid:22707770 PMCid:PMC3371522

10. Talib NH, Leelavathi M, Hamzah Z. Common adverse cutaneous drug reaction patterns and the causative drugs in Malaysia, South African Family Practice. 2015; 57:4, 227-30. https:// doi.org/10.1080/20786190.2015.1024026

11. Bigby M. Rates of cutaneous reactions to drugs. Arch Dermatol. 2001;137(6):765-70. [sdoi.org/10.1001/archderm.137.12.1639

12. Grando LR, Schmitt TA, Bakos RM. Severe cutaneous reactions to drugs in the setting of a general hospital. An Bras Dermatol. 2014; 89 (5):758-62. https://doi.org/10.1590/abd18064841.20142997 PMid:25184915 PMCid:PMC4155954.

Citation: Ghimire RB, Bhattarai S, Pokharel, Aryal E, Shrestha PR. Clinical Profile of Severe Cutaneous Drug Eruptions in a Tertiary Care Hospital. JCMS Nepal. 2019; 15(3):156-9. 